Jane Hodgkinson, John Saffell, Jonathan Luff, John Shaw, Jeremy Ramsden, Carlos Huggins, Robert Bogue and Roger Carline

\title{
Gas sensors 1. The basic technologies and applications
}

ABSTRACT. This paper focuses on the technologies used for gas sensing, notably optical, electrical, mass-based and biological. Applications are summarized under the headings of healthcare, security, environment and processes, as a precursor to the more detailed consideration of gas sensor markets in Part 2, the following paper.

Nanotechnology Perceptions 5 (2009) 71-82

Nonsubscribers: purchase individual article 